\title{
Substituted Quinazolines, 1. Synthesis and Antitumor Activity of Certain Substituted 2-Mercapto-4(3H)-quinazolinone Analogs.
}

\author{
S.G. Abdel Hamid*, H.A. El-Obeid, K.A. Al-Rashood, A.A. Khalil and \\ H.I. El-Subbagh.* \\ Department of Pharmaceutical Chemistry, College of Pharmacy, \\ King Saud University, P.O. Box 2457, Riyadh-11451 \\ Saudi Arabia.
}

\begin{abstract}
A new series of $4(3 H)$-quinazolinone analogs bearing 6-iodo and 2-thioether functions were synthesized and screened for their in vitro antitumor activity. Eight compounds were identified as active anticancer agents. 2-Mercapto-3-benzyl-4-thioxo-6-iodo-3H-quinazoline (2) and 2-(2,4dinitrophenyl)-3-benzyl-6-iodo-4-(3H)-quinazolinone (9) proved to be the most active compounds in this study. They showed MG-MID GI 50 , TGI, $\mathrm{LC}_{50}$ values of $3.9,25.2,82.3$ and $2.7,12.3,38.7 \mu \mathrm{M}$, respectively. The detailed synthesis and biological screening data are reported.
\end{abstract}

(Keywords: Synthesis, 4(3H)-quinazolinone, Antitumor testing).

\section{Introduction}

Quinazolines have been reported to be biologically versatile compounds possessing variety of activity including anticancer potency. ${ }^{1}$ An extensive interest in quinazolines has been increased since the discovery of raltitrexed (A) and thymitaq (B) and their activity as thymidylate enzyme inhibitors. ${ }^{2,3}$ Overexpression of the epidermal growth factor receptor (EGFR) tyrosine kinase is associated with poor prognosis in a significant proportion of human tumors. ${ }^{4.5}$ 4-Anilinoquinazolines proved to inhibit EGFR autophosphorylation and EGF-stimulated signal transduction and considered as a new class of anticancer drugs. ${ }^{6-13}$

Quinazoline analogs also showed a remarkable activity against the opportunistic infections of Pneumocystis carinii and Toxoplasma gondii through the inhibition of dihydrofolate reductase 
<smiles>Cc1ccc2nc(N)[nH]c(=O)c2c1Sc1ccncc1</smiles>

Raltitrexed, A<smiles>CN(Cc1ccc2nc(N)[nH]c(=O)c2c1)c1ccc(C(=O)N[C@@H](CCC(=O)O)C(=O)O)s1</smiles>

Thymitaq, B

enzyme. Those microorganisms proved to be the principal cause of death in patients with immunocompromised diseases such as Acquired Immunodeficiency Syndrome (AIDS). ${ }^{14-17}$

Enzyme-mediated repair of single- or double- strand lesions in DNA is an established mechanism of resistance to antitumor DNA-damaging drugs and radiotherapy. ${ }^{18,19}$ Quinazolines proved to inhibit this DNA repair enzymes and thus a new strategy for the potentiation of DNAdamaging anticancer therapies is obtained. ${ }^{20}$

In the present study, a new series of 2-substituted mercapto-3-benzyl-6-iodo-4(3H)quinazolinone was designed and synthesized, in such a fashion that the 5-thioether function of $\mathbf{A}$ was moved to position 2-. Thioether, ${ }^{21} \alpha, \beta$-unsaturated ketone, ${ }^{22}$ amide $^{23}$ and 1,3-isoindoledione ${ }^{24}$ are functional groups known to enhance the antitumor activity. Those functions, in addition to others such as alkyl, cycloalkyl, alkyl esters, arylalkyl, aryl and heteroaryl were combined with $4(3 H)$-quinazolinone heterocycle via a thioether linkage at position 6-. The objective of forming these hybrids, is an attempt to reach an active antitumor agent with potentiated activity towards cancerous cells and less toxicity towards normal cells.

\section{Results and Discussion}

\section{Chemistry}

The synthetic strategy to synthesize the target compounds $\mathbf{2 - 2 5}$, is depicted in schemes 1-3. 


\section{Scheme 1:}<smiles>S=c1c2cc(I)ccc2nc(S)n1Cc1ccccc1</smiles>

2

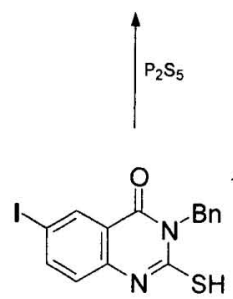<smiles>[R]Sc1nc2ccc(I)cc2c(=O)n1Cc1ccccc1</smiles>

3: $\mathrm{R}=\mathrm{C}_{2} \mathrm{H}_{5} ; 4: \mathrm{R}=\mathrm{CH}\left(\mathrm{CH}_{3}\right)_{2}$ 5: $\mathrm{R}=\mathrm{CH}(\mathrm{Ph})_{2} ; \mathbf{6}: \mathrm{R}=\mathrm{C}_{6} \mathrm{H}_{11}$

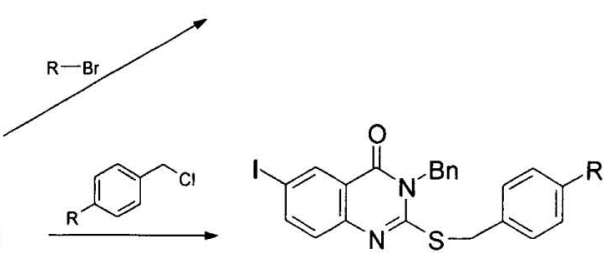

7: $\mathrm{R}=\mathrm{Cl} ; \mathbf{8}: \mathrm{R}=\mathrm{Br}$<smiles>[R]C(CC(=O)OCC)Sc1nc2ccc(I)cc2c(=O)n1Cc1ccccc1</smiles>

11: $R=H, n=1$

12: $R=H, n=2$

13: $\mathrm{R}=\mathrm{CH}_{3}, \mathrm{n}=1$

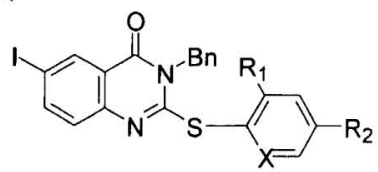

9: $R_{1}=R_{2}=N_{2} ; X=C H$

10: $R_{1}=N_{2} ; R_{2}=H ; X=N$

The starting material, 2-mercapto-3-benzyl-6-iodo-4(3H)-quinazolinone (1) ${ }^{25}$ was treated with $\mathrm{P}_{2} \mathrm{~S}_{5}$ to afford the 4-thioxo derivative $\mathbf{2}$ in quantitative yield. The 2-mercapto function of $\mathbf{1}$ was alkylated using variety of alkyl halides such as ethyl, isopropyl, benzhydryl and cyclohexyl bromide to give the 2-alkylthio analogs 2-6. Meanwhile, the 2-mercapto function of 1 was benzylated using either 4-chloro- or 4-bromobenzyl bromide to produce the 4-substituted benzylthio-derivatives 7 and 8 . Treatment of 1 with chloronitrobenzene or chloronitropyridine gave the arylthio compounds 9 and 


\section{Scheme 2:}<smiles>CC(=O)CSc1nc2ccc(I)cc2c(=S)n1Cc1ccccc1</smiles>

14

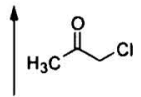<smiles>O=c1c2cc(I)ccc2nc(S)n1Cc1ccccc1</smiles><smiles>O=C(CCl)Nc1ccc(Br)cc1</smiles><smiles>[R]c1ccc(NC(=O)CSc2nc3ccc(I)cc3c(=O)n2Cc2ccccc2)cc1</smiles>

20: $\mathrm{R}=\mathrm{H} ; 2$ 1: $\mathrm{R}=\mathrm{Cl}$

22: $\mathrm{R}=\mathrm{Br}$

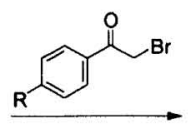<smiles>[R]c1ccc(C(=O)CSc2nc3ccc(I)cc3c(=O)n2Cc2ccccc2)cc1</smiles>

16: $\mathrm{R}=\mathrm{H} ; 17: \mathrm{R}=\mathrm{Cl}$;

18: $R=F ; 19: R=\mathrm{NO}_{2}$

10. ${ }^{25}$ Reacting 1 with either ethyl bromoacetate, ethyl 2-bromopropionate or ethyl 3-bromopropionate gave the thioalkyl esters $\mathbf{1 1 - 1 3 ^ { 2 5 }}$ (Scheme 1, Table 1). Reacting $\mathbf{1}$ with chloroacetone gave the 2-oxo-propylthio- analog 14 which was subsequently reacted with benzaldehyde to give the corresponding $\alpha, \beta$-unsaturated ketone derivative 15 . Treatment of 1 with 4-substituted phenacyl bromides and $2^{\prime}$-chloro-4-substituted-acetanilide afforded the targets 16-18 and 20-22, respectively (Scheme 2, Table 1). Oxidation of 1 using iodine solution in alkaline 
Scheme 3:

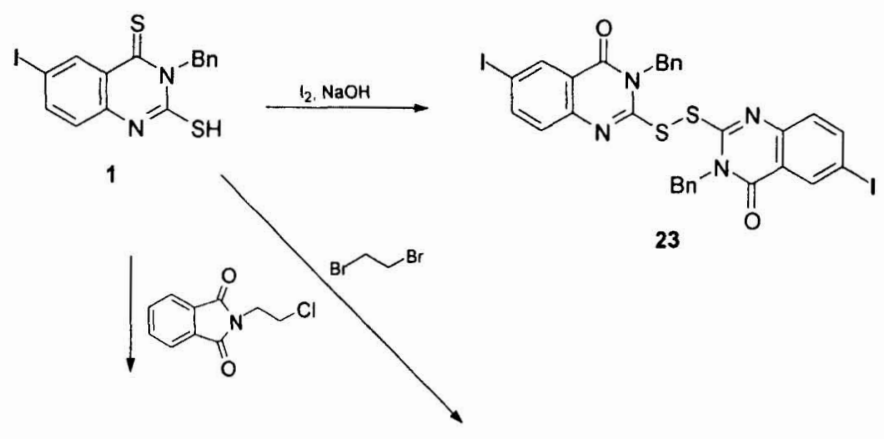<smiles>O=C1c2ccccc2C(=O)N1CCSc1nc2ccc(I)cc2c(=O)n1Cc1ccccc1</smiles>

25<smiles></smiles>

medium produced the disulphide analog 23. Reacting two moles of 1 with one mole of 1,2-dibromoethane afforded the bis- compound $\mathbf{2 4}$, while its reaction with $\mathrm{N}$-(2-chloroethyl)phthalimide gave the target 1,3-isoindoledione analog 25 (Scheme 3, Table 1).

\section{Antitumor Testing}

The synthesized compounds were subjected to the NCI's in vitro, one dose primary anticancer assay, using a 3-cell line panel consisting of MCF 7 (breast), NCI-H460 (lung) and SF-268 (CNS) cancers. Compounds which reduce the growth of any one of the cell lines to $32 \%$ or less are passed on for evaluation in the full panel of 60 cell lines over a 5 -log dose range. ${ }^{26,27}$ Three response parameters, median growth inhibition $\left(\mathrm{GI}_{50}\right)$, total growth inhibition (TGI), and median lethal concentration $\left(\mathrm{LC}_{50}\right)$ were calculated for each cell line. The NCI antitumor drug discovery 
Table 1: Physicochemical properties and primary antitumor activity of the synthesized compounds.

\begin{tabular}{|c|c|c|c|c|c|}
\hline Compd & Solvent & Yield (\%) & $\mathrm{MP}\left({ }^{\circ} \mathrm{C}\right)$ & Molecular formulae & Primary anticancer assay \\
\hline 1 & EtOH, benzene & 60 & $250-1$ & Ref. 25 & $a$ \\
\hline 2 & Xylene & 70 & $266-7$ & $\mathrm{C}_{15} \mathrm{H}_{11} \mathrm{IN}_{2} \mathrm{~S}_{2}$ & $a$ \\
\hline 3 & EtOH, benzene & 75 & $94-5$ & $\mathrm{C}_{17} \mathrm{H}_{15} \mathrm{IN}_{2} \mathrm{OS}$ & $b$ \\
\hline 4 & EtOH & 65 & $155-7$ & $\mathrm{C}_{18} \mathrm{H}_{17} \mathrm{IN}_{2} \mathrm{OS}$ & $b$ \\
\hline 5 & $\mathrm{EtOH}$ & 70 & $165-7$ & $\mathrm{C}_{28} \mathrm{H}_{21} \mathrm{IN}_{2} \mathrm{OS}$ & $b$ \\
\hline 6 & $\mathrm{EtOH}$ & 40 & $260-1$ & $\mathrm{C}_{21} \mathrm{H}_{21} \mathrm{IN}_{2} \mathrm{OS}$ & $b$ \\
\hline 7 & $\mathrm{EtOH}$ & 50 & $175-7$ & $\mathrm{C}_{22} \mathrm{H}_{16} \mathrm{ClIN}_{2} \mathrm{OS}$ & $b$ \\
\hline 8 & EtOH & 55 & $225-7$ & $\mathrm{C}_{22} \mathrm{H}_{16} \mathrm{BrIN}_{2} \mathrm{OS}$ & $n t$ \\
\hline 9 & DMF & 70 & $243-4$ & Ref. 25 & $a$ \\
\hline 10 & $\mathrm{BuOH}$ & 71 & $286-7$ & Ref. 25 & $a$ \\
\hline 11 & $\mathrm{MeOH}$ & 70 & $125-7$ & Ref. 25 & $n t$ \\
\hline 12 & $\mathrm{MeOH}$ & 60 & $100-2$ & Ref. 25 & $a$ \\
\hline 13 & $\mathrm{MeOH}, \mathrm{H}_{2} \mathrm{O}$ & 75 & $108-10$ & Ref. 25 & $n t$ \\
\hline 14 & $\mathrm{EtOH}, \mathrm{H}_{2} \mathrm{O}$ & 50 & $246-8$ & Ref. 25 & $b$ \\
\hline 15 & $\mathrm{EtOH}$ & 40 & $215-7$ & $\mathrm{C}_{25} \mathrm{H}_{19} \mathrm{IN}_{2} \mathrm{O}_{2} \mathrm{~S}$ & $a$ \\
\hline 16 & $\mathrm{MeOH}$ & 50 & $180-2$ & $\mathrm{C}_{23} \mathrm{H}_{17} \mathrm{IN}_{2} \mathrm{O}_{2} \mathrm{~S}$ & $b$ \\
\hline 17 & $\mathrm{EtOH}$ & 61 & $210-2$ & $\mathrm{C}_{23} \mathrm{H}_{16} \mathrm{ClIN}_{2} \mathrm{O}_{2} \mathrm{~S}$ & $b$ \\
\hline 18 & Dioxane & 65 & $176-8$ & $\mathrm{C}_{23} \mathrm{H}_{16} \mathrm{FIN}_{2} \mathrm{O}_{2} \mathrm{~S}$ & $n t$ \\
\hline 19 & $\mathrm{EtOH}$ & 45 & $258-260$ & $\mathrm{C}_{23} \mathrm{H}_{16} \mathrm{IN}_{3} \mathrm{O}_{4} \mathrm{~S}$ & $b$ \\
\hline 20 & EtOH, Dioxane & 50 & $234-6$ & $\mathrm{C}_{23} \mathrm{H}_{18} \mathrm{IN}_{3} \mathrm{O}_{2} \mathrm{~S}$ & $a$ \\
\hline 21 & EtOH, Dioxane & 60 & $222-4$ & $\mathrm{C}_{23} \mathrm{H}_{17} \mathrm{ClIN}_{3} \mathrm{O}_{2} \mathrm{~S}$ & $b$ \\
\hline 22 & MeOH, Benzene & 55 & $225-7$ & $\mathrm{C}_{23} \mathrm{H}_{17} \mathrm{BrIN}_{3} \mathrm{O}_{2} \mathrm{~S}$ & $a$ \\
\hline 23 & $\mathrm{CHCl}_{3}$, Hexane & 60 & $137-9$ & $\mathrm{C}_{30} \mathrm{H}_{20} \mathrm{I}_{2} \mathrm{~N}_{4} \mathrm{O}_{2} \mathrm{~S}_{2}$ & $b$ \\
\hline 24 & $\mathrm{AcOH}$ & 50 & $265-7$ & $\mathrm{C}_{32} \mathrm{H}_{24} \mathrm{I}_{2} \mathrm{~N}_{4} \mathrm{O}_{2} \mathrm{~S}_{2}$ & $b$ \\
\hline 25 & $\mathrm{AcOH}$ & 40 & $258-9$ & $\mathrm{C}_{25} \mathrm{H}_{18} \mathrm{IN}_{3} \mathrm{O}_{3} \mathrm{~S}$ & $b$ \\
\hline
\end{tabular}

$a$, Compound which reduces the growth of any one of the cell lines NCI-H460 (lung), SF-268 (CNS) and MCF7 (breast) to $32 \%$ or less at concentration of $100 \mu \mathrm{M}$, are passed on for evaluation in the full panel of 60 cell lines. $b$, inactive compound. $n t$, compound not tested.

screen has been designed to distinguish between broad-spectrum antitumor and tumor or subpanelselective compounds. ${ }^{28}$

In the present study, compounds $1,2,9,10,12,15,20$ and 22 passed the primary anticancer at an arbitrary concentration of $100 \mu \mathrm{M}$ (Table 1). Consequently, those active compounds were carried over and tested against a panel of 60 different tumor cell lines. The eight tested quinazoline analogs showed a distinctive potential pattern of selectivity as well as broad-spectrum antitumor 
Table 2: Growth inhibitory concentrations $\left(\mathrm{GI}_{50}\right.$, TGI and $\left.\mathrm{LC}_{50}\right)$ of some selected in vitro tumor cell lines $(\mu \mathrm{M}){ }^{a}$

\begin{tabular}{|c|c|c|c|c|c|c|}
\hline \multirow[t]{2}{*}{ Compd $^{h}$} & \multirow[t]{2}{*}{ Activity } & \multicolumn{3}{|c|}{ Leukemia } & \multicolumn{2}{|c|}{ Renal } \\
\hline & & CCRF-CEM & HL-60 (TB) & MOLT-4 & บo-31 & $786-O$ \\
\hline \multirow[t]{3}{*}{1} & $\mathrm{GI}_{50}$ & 5.7 & 6.8 & $c$ & 0.5 & 11.5 \\
\hline & TGI & 23.1 & 28.5 & $c$ & 1.8 & 63.0 \\
\hline & $\mathrm{LC}_{50}$ & 82.3 & 82.3 & $c$ & 46.5 & $c$ \\
\hline \multirow[t]{3}{*}{9} & $\mathrm{GI}_{50}$ & 0.2 & 1.8 & 1.6 & 2.2 & 0.9 \\
\hline & TGI & $c$ & $5 . .3$ & $c$ & 4.1 & 2.3 \\
\hline & $\mathrm{LC}_{50}$ & $c$ & $c$ & $c$ & 7.6 & 5.2 \\
\hline \multirow[t]{3}{*}{10} & $\mathrm{GI}_{50}$ & 0.3 & 0.1 & 23.2 & 14.3 & 23.2 \\
\hline & TGI & $c$ & 2.0 & $c$ & $c$ & $c$ \\
\hline & $\mathrm{LC}_{50}$ & $c$ & $c$ & $c$ & $c$ & $c$ \\
\hline \multirow[t]{3}{*}{12} & $\mathrm{GI}_{50}$ & 13.9 & 21.8 & $<0.01$ & 1.3 & 23.4 \\
\hline & TGI & 83.3 & $c$ & $<0.01$ & 2.6 & $c$ \\
\hline & $\mathrm{LC}_{50}$ & $c$ & $c$ & 3.4 & 5.6 & $c$ \\
\hline \multirow[t]{3}{*}{15} & $\mathrm{GI}_{50}$ & 13.8 & 16.9 & 10.3 & 0.2 & 13.3 \\
\hline & TGI & $c$ & 50.3 & 39.2 & 1.0 & 32.4 \\
\hline & $\mathrm{LC}_{50}$ & $c$ & $c$ & $c$ & 3.8 & 78.6 \\
\hline \multirow[t]{3}{*}{20} & $\mathrm{GI}_{50}$ & $c$ & $c$ & $c$ & 0.3 & 8.6 \\
\hline & TGI & $c$ & $c$ & $c$ & 1.6 & 23.9 \\
\hline & $\mathrm{LC}_{50}$ & $c$ & $c$ & $c$ & 16.4 & 61.8 \\
\hline \multirow[t]{3}{*}{22} & $\mathrm{GI}_{50}$ & $c$ & $c$ & $c$ & 0.1 & 58.7 \\
\hline & TGI & $c$ & $c$ & $c$ & 0.4 & $c$ \\
\hline & $\mathrm{LC}_{50}$ & $c$ & $c$ & $c$ & 1.7 & $c$ \\
\hline
\end{tabular}

${ }^{a}$ Data obtained from NCl's in vitro disease oriented human tumor cell screen (see references 26-28 for details), ${ }^{b}$ compound 2 showed activity $>100 \mu \mathrm{M}$ against these cell lines. ${ }^{c} \mathrm{GI}_{50}$, TGI and $\mathrm{LC}_{50}$ values $>100 \mu \mathrm{M}$.

activity. With regard to sensitivity against individual cell lines, compounds 9 and 10 showed $\mathrm{GI}_{50}$ effectiveness against leukemia CCRF-CEM cell line at concentrations of 0.2 and $0.3 \mu \mathrm{M}$, respectively. Compound 10 also showed a remarkable activity against HL-60 (TB) leukemia cell line at $\mathrm{GI}_{50}$ and TGI levels with 0.1 and $2.0 \mu \mathrm{M}$ concentrations, respectively. MOLT-4 leukemia 
Table 3: Median growth inhibitory concentration $\left(\mathrm{GI}_{50}, \mu \mathrm{M}\right)$ total growth inhibitory concentration $(\mathrm{TGI}, \mu \mathrm{M})$ and median lethal concentration $\left(\mathrm{LC}_{50}, \mu \mathrm{M}\right)$ of in vitro subpanel tumor cell lines.

\begin{tabular}{|c|c|c|c|c|c|c|c|c|c|c|c|c|}
\hline \multirow{2}{*}{ Compd } & \multicolumn{9}{|c|}{ Subpanel tumor cell lines ${ }^{a}$} & \multicolumn{3}{|c|}{ MG-MID ${ }^{h}$} \\
\hline & I & II & III & IV & V & VI & VII & VIII & IX & $\mathrm{GI}_{50}$ & TGI & $\mathbf{L C}_{50}$ \\
\hline 1 & 30.9 & 18.1 & 37.0 & 15.6 & 20.3 & 19.0 & 10.2 & 17.0 & 36.0 & 14.6 & 70.7 & 92.3 \\
\hline 2 & 2.9 & 3.7 & 7.2 & 5.0 & 3.4 & 6.2 & 3.7 & 3.0 & 4.5 & 3.9 & 25.2 & 82.3 \\
\hline 9 & 1.0 & 4.7 & 2.7 & 4.8 & 2.8 & 6.1 & 4.7 & 2.6 & 2.5 & 2.7 & 12.3 & 38.7 \\
\hline 10 & 14.7 & 27.7 & 26.7 & 33.8 & 33.2 & 43.9 & 24.8 & 24.5 & 19.4 & 20.6 & 89.4 & $\mathrm{~d}$ \\
\hline 12 & 8.0 & 21.7 & 26.9 & 27.0 & 36.5 & 50.8 & 22.2 & 43.6 & 32.6 & 20.1 & 66.3 & 84.7 \\
\hline 15 & 11.3 & 17.2 & 23.4 & 27.7 & 42.9 & 35.4 & 10.9 & 22.9 & 31.3 & 17.5 & 72.1 & 92.3 \\
\hline 20 & 73.2 & 20.3 & 78.4 & 10.2 & 33.9 & 16.6 & 13.3 & 20.7 & 40.7 & 20.9 & 58.8 & 92.3 \\
\hline 22 & 72.7 & 56.3 & 76.7 & 32.0 & 61.4 & 47.5 & 37.6 & 44.0 & 61.0 & 37.1 & 72.1 & 89.4 \\
\hline
\end{tabular}

${ }^{a} \mathrm{GI}_{50}$ values against I, leukemia; II, non-small cell lung cancer; III, colon cancer; IV, CNS cancer; V, melanoma; VI, ovarian cancer; VII, renal cancer; VIII, prostate cancer; IX, breast cancer cell lines. ${ }^{b} \mathrm{GI}_{50}$, TGI and $\mathrm{LC}_{50}$ full panel mean-graph mid point $(\mu \mathrm{M})$.

cell line proved to be sensitive against compound 12 with $\mathrm{GI}_{50}$, TGI and $\mathrm{LC}_{50}$ concentrations of $<0.01,<0.01$ and $3.4 \mu \mathrm{M}$, respectively. UO-31 renal cell line proved to be sensitive against compounds $1,15,20$ and 22 with $\mathrm{GI}_{50}$ concentrations of $0.5,0.2,0.3$ and $0.1 \mu \mathrm{M}$, respectively. Compound 9 showed an activity against 786-0 renal cell line at $\mathrm{GI}_{50}$ level with concentration of 0.9 $\mu \mathrm{M}$ (Table 2).

With regard to broad-spectrum antitumor activity, compounds $1,2,9,12,15,20$ and 22 showed $\mathrm{GI}_{50}$, TGI and $\mathrm{LC}_{50}(\mathrm{MG}-\mathrm{MID})<100 \mu \mathrm{M}$ against leukemia, non-small-cell lung, colon, CNS, melanoma, ovarian, renal, prostate and breast cancer subpanel cell lines. Compound 10 showed (MG-MID) values $<100 \mu \mathrm{M}$ at only the $\mathrm{GI}_{50}$ and TGI levels. Compound 22 is the least effective member of those eight compounds with $\mathrm{GI}_{50}$, TGI, $\mathrm{LC}_{50}$ values of $37.1,72.1,89.4 \mu \mathrm{M}$, respectively (Table 3 ). 
Structure activity correlation of the synthesized compounds showed that 2-mcerapto-3benzyl-6-iodo-4 $(3 H)$-quinazolinone (1) proved to possess a broad-spectrum antitumor activity at MG-MID GI ${ }_{50}$, TGI and $\mathrm{LC}_{50}$ values of $14.6,70.7$ and $92.3 \mu \mathrm{M}$, respectively. Thiation of 1 produced the 4-thioxo analog 2 , which proved to be almost four times more active than $\mathbf{1}$. Alkylation of the 2-mercapto function of 1 with alkyl $(2,4)$ cycloalkyl $(6)$ or arylalkyl $(5,7)$ produced inactive compounds; while alkylation with alkylesters (12) preserved the activity. Reacting 1 with 2,4-dinitrochlorobenzene gave the thioether 9 with almost five folds increase in the antitumor activity $\left(\mathrm{GI}_{50}, \mathrm{TGI}\right.$ and $\mathrm{LC}_{50}$ values of $2.7,12.3$ and $38.7 \mu \mathrm{M}$, respectively), replacing the nitrobenzene moiety of 9 by nitropyridine (10) decreased the magnitude of activity dramatically. Conversion of the inactive alkylester 11 into its corresponding amides 20 and 22 increased the antitumor activity. Introduction of the $\alpha, \beta$-unsaturated moiety to the 2-mercapto function of 1 produced the target $\mathbf{1 5}$ with a little increase in the activity. Formation of the bis- compounds $\mathbf{2 3}$ and 24 or the introduction of the 1,3-isoindoledione moiety (25) produced inactive analogs.

In conclusion, compounds 2-mercapto-3-benzyl-4-thioxo-6-iodo-3H-quinazoline (2) and 2(2,4-dinitrophenyl)-3-benzyl-6-iodo-4-(3H)-quinazolinone (9) proved to be the most active members in this series. These two quinazolinone analogs could be considered as useful template for future development to obtain more potent antitumor agents.

\section{Experimental}

Melting points were determined on a Mettler FP80 melting point apparatus and are uncorrected. Microanalyses were performed on a Perkin-Elmer 240 elemental analyzer at the Central Research Laboratory, College of Pharmacy, King Saud University. All of the new compounds were analyzed for $\mathrm{C}, \mathrm{H}$ and $\mathrm{N}$ and agreed with the proposed structures within $\pm 0.4 \%$ of 
the theoretical values. ${ }^{1} \mathrm{H}$ NMR spectra were recorded on a Varian XL, $400 \mathrm{MHz}$ FT spectrometer; chemical shifts are expressed in $\delta$ ppm with reference to TMS. Mass spectral data were obtained on a Shimadzu GC/MS QP 5000 apparatus. IR spectra were performed on Pye Unicum Sp 1100. Thinlayer chromatography was performed on precoated $(0.25 \mathrm{~mm})$ silica gel plates; compounds were detected with a $254-\mathrm{nm}$ UV lamp. Silica gel (60-230 mesh) was employed for routine column chromatography separations. The synthesis of compounds $1,9-14^{25}$ were previously reported. The synthesized compounds were tested in vitro for their antitumor activity at the $\mathrm{NCl}$, Bethesda, MD, USA.

\section{3-Benzyl-6-iodo-2-mercapto-4-thioxo-3H-quinazoline 2:}

2-Mercapto-3-benzyl-6-iodo-4-(3H)-quinazolinone $(1,3.9 \mathrm{~g}, 0.01 \mathrm{~mol})$ and $\mathrm{P}_{2} \mathrm{~S}_{5}(5.0 \mathrm{~g})$ in pyridine $(50 \mathrm{ml})$ was heated under reflux for $5 \mathrm{~h}$. Solvent was evaporated under reduced pressure and the obtained residue was washed with dil. $\mathrm{HCl}$, water and recrystallized from xylene (Table 1). ${ }^{1} \mathrm{H}$ NMR (DMSO-d 6 ), $\delta 3.4$ (brs, 1H, SH), 5.12 (s, 2H, $\mathrm{CH}_{2} \mathrm{Ph}$ ), 7.25-7.36 (m, 6H, ArH), 8.06 (d, $J=15 \mathrm{~Hz}, 1 \mathrm{H}, \mathrm{ArH}), 8.34$ (s, 1H, ArH). m/z (410, 30\%); $\operatorname{IR}_{\mathrm{KBr}} \mathrm{cm}^{-1}(3080,3060,2930,2560,1601$, $1495,1455,1150)$.

\section{2-Alkylthio-3-benzyl-6-iodo-4(3H)-quinazolinones 3-6:}

To a solution of $1(3.9 \mathrm{~g}, 0.01 \mathrm{~mol})$ in acetone $(50 \mathrm{ml})$, anhydrous $\mathrm{K}_{2} \mathrm{CO}_{3}(2.0 \mathrm{~g})$ was added followed by either ethylbromide, isopropyl bromide, benzyhydryl bromide or cyclohexyl bromide $(0.015 \mathrm{~mol})$. The reaction mixture was heated under reflux for $20 \mathrm{~h}$. The solvent was removed in vacuo and the obtained solid was recrystallized from the appropriate solvent (Table 1). 'H NMR (DMSO-d $\left.\mathrm{d}_{6}\right), 3: \delta 1.10$ (t, $\left.J=7 \mathrm{~Hz}, 3 \mathrm{H}, \mathrm{CH}_{3} \mathrm{CH}_{2}\right), 3.42\left(\mathrm{q}, J=7 \mathrm{~Hz}, 2 \mathrm{H}, \mathrm{CH}_{3} \mathrm{CH}_{2}\right), 5.41(\mathrm{~s}, 2 \mathrm{H}$, $\left.\mathrm{CH}_{2} \mathrm{Ph}\right), 7.24-7.32(\mathrm{~m}, 6 \mathrm{H}, \mathrm{ArH}), 8.12(\mathrm{~s}, 1 \mathrm{H}, \mathrm{ArH}), 8.32$ (s, $\left.1 \mathrm{H}, \mathrm{ArH}\right) . \mathrm{m} / \mathrm{z}(422,28 \%) ; \mathrm{IR}_{\mathrm{KBr}} \mathrm{cm}^{-1}$ 
$(3070,3040,2980,2950,1695,1601,1495,1455,1200,700) 4: \delta 0.9\left(\mathrm{~d}, J=15 \mathrm{~Hz}, 6 \mathrm{H}, \mathrm{CH}\left(\mathrm{CH}_{3}\right)_{2}\right)$, 3.2-3.5 (m, $\left.1 \mathrm{H}, \mathrm{CH}\left(\mathrm{CH}_{3}\right)_{2}\right), 5.50\left(\mathrm{~s}, 2 \mathrm{H}, \mathrm{CH}_{2} \mathrm{Ph}\right), 7.22-7.33(\mathrm{~m}, 6 \mathrm{H}, \mathrm{ArH}), 8.05(\mathrm{~s}, 1 \mathrm{H}, \mathrm{ArH}), 8.30$ (s, 1H, ArH). m/z $(436,15 \%) \mathrm{IR}_{\mathrm{KBr}} \mathrm{cm}^{-1}(3060,3040,2930,2880,1695,1601,1495,1455,1200$, 700). 5: $\delta 4.6\left(\mathrm{~s}, 1 \mathrm{H}, \mathrm{CH}(\mathrm{Ph})_{2}\right), 5.52\left(\mathrm{~s}, 2 \mathrm{H}, \mathrm{CH}_{2} \mathrm{Ph}\right), 7.25-7.62(\mathrm{~m}, 16 \mathrm{H}, \mathrm{ArH}), 8.04(\mathrm{~s}, 1 \mathrm{H}, \mathrm{ArH})$, $8.32(\mathrm{~s}, 1 \mathrm{H}, \mathrm{ArH}) . \mathrm{m} / \mathrm{z}(560,30 \%) ; \mathrm{IR}_{\mathrm{KBr}} \mathrm{cm}^{-1}(3070,3050,2930,1695,1601,1495,1400,1200$, 750). 6: $\delta$ 0.9-2.1 (m, 11H, cyclohexyl), $5.49\left(\mathrm{~s}, 2 \mathrm{H}, \mathrm{CH}_{2} \mathrm{Ph}\right), 7.23-7.32(\mathrm{~m}, 6 \mathrm{H}, \mathrm{ArH}), 8.06(\mathrm{~s}, 1 \mathrm{H}$, $\operatorname{ArH}), 8.29$ (s, 1H, ArH). m/z $(476,10 \%) ; \mathrm{IR}_{\mathrm{KBr}} \mathrm{cm}^{-1}(3060,3040,2980,2890,2850,1695,1600$, $1495,1455,1200,780)$.

\section{3-Benzyl-6-iodo-2-(substituted phenylmethylthio)-4-(3H)-quinazolinone 7, 8:}

A mixture of 1 (3.9 g, $0.01 \mathrm{~mol})$, 4-substituted-benzyl bromide $(0.015 \mathrm{~mol})$ and anhydrous $\mathrm{K}_{2} \mathrm{CO}_{3}(2.0 \mathrm{~g})$ in acetone $(50 \mathrm{ml})$ was heated under reflux for $12 \mathrm{~h}$. The reaction mixture was filtered while hot, the filtrate was evaporated in vacuo and the obtained solid was recrystallized from the appropriate solvent (Table 1). ${ }^{1} \mathrm{H} \mathrm{NMR}\left(\mathrm{CDCl}_{3}\right) 7: \delta 5.21\left(\mathrm{~s}, 2 \mathrm{H}, \mathrm{CH}_{2} \mathrm{Ph}\right), 5.52(\mathrm{~s}, 2 \mathrm{H}$, $\left.\mathrm{CH}_{2} \mathrm{Ph}\right), 7.19-7.59$ (m, 10H, ArH), 8.02 (d, $\left.J=15 \mathrm{~Hz}, 1 \mathrm{H}, \mathrm{ArH}\right), 8.32$ (d, $\left.J=15 \mathrm{~Hz}, 1 \mathrm{H}, \mathrm{ArH}\right) . \mathrm{m} / \mathrm{z}$ (520, 10\%), (518, 29\%); $\operatorname{IR}_{\mathrm{KBr}} \mathrm{cm}^{-1}(3060,3030,2930,1690,1600,1490,1450,1150,1090,700)$. 8: $\delta 5.23\left(\mathrm{~s}, 2 \mathrm{H}, \mathrm{CH}_{2} \mathrm{Ph}\right), 5.49\left(\mathrm{~s}, 2 \mathrm{H}, \mathrm{CH}_{2} \mathrm{Ph}\right), 7.16-7.61(\mathrm{~m}, 10 \mathrm{H}, \mathrm{ArH}), 8.04(\mathrm{~s}, 1 \mathrm{H}, \mathrm{ArH}), 8.34(\mathrm{~s}$, $1 \mathrm{H}, \mathrm{ArH}) . \mathrm{m} / \mathrm{z}(564,21 \%),(562,22 \%), \mathrm{IR}_{\mathrm{KBr}} \mathrm{cm}^{-1}(3075,3035,2940,1695,1600,1495,1450$, $1155,1050,700)$.

\section{3-Benzyl-6-iodo-2-[(4-phenyl-2-oxo-3-propen-1-yl)thio]- -4(3H)-quinazolinone 15:}

A solution of 2-(2-oxo-propylthio)-3-benzyl-6-iodo-4(3H)-quinazolinone $(14,4.5 \mathrm{~g}, 0.01$ $\mathrm{mol})$ and $\mathrm{NaOEt}(0.8 \mathrm{~g}, 0.012 \mathrm{~mol})$ in ethanol $(50 \mathrm{ml})$ was stirred at room temperature for $2 \mathrm{~h}$. 
Benzaldehyde $(1.2 \mathrm{~g}, 0.012 \mathrm{~mol})$ in ethanol $(20 \mathrm{ml})$ was added dropwise and stirring was continued for another $24 \mathrm{~h}$. The reaction mixture was adjusted to $\mathrm{pH} 6$ using dil. $\mathrm{HCl}$, the precipitated solid was filtered, dried and recrystalized from ethanol (Table 1). ${ }^{1} \mathrm{H}$ NMR (DMSO- $\mathrm{d}_{6}$ ): $\delta 4.30$ (s, $2 \mathrm{H}$, $\left.\mathrm{CH}_{2} \mathrm{CO}\right), 5.83\left(\mathrm{~s}, 2 \mathrm{H}, \mathrm{CH}_{2} \mathrm{Ph}\right), 6.56(\mathrm{~d}, J=10 \mathrm{~Hz}, 1 \mathrm{H}$, olefinic $\mathrm{H}), 7.21-7,63(\mathrm{~m}, 12 \mathrm{H}$, olefinic and $\operatorname{ArH}), 8.05$ (d, $J=15 \mathrm{~Hz}, 1 \mathrm{H}, \operatorname{ArH}), 8.33$ (s, 1H, ArH). m/z $(538,10 \%) \operatorname{IR}_{\mathrm{KBr}} \mathrm{cm}^{-1}(3065,3035$, $2980,1695,1600,1485,1450,1200,700)$.

\section{3-Benzyl-6-iodo-2-(substituted phenylcarbonylmethylthio)-4(3H)-quinazolinones 16-19:}

A mixture of $1(3.9 \mathrm{~g}, 0.01 \mathrm{~mol})$, the appropriate 4-substituted phenacyl bromide $(0.01 \mathrm{~mol})$ and anhydrous $\mathrm{K}_{2} \mathrm{CO}_{3}(2.0 \mathrm{~g})$ in acetone $(50 \mathrm{ml})$ was heated under reflux for $24 \mathrm{~h}$. The reaction mixture was filtered while hot and the filtrate was concentrated under reduced pressure, then cooled. The obtained solid was filtered, dried and recrystallized from the suitable solvent (Table 1). ${ }^{1} \mathrm{H}$ NMR $\left(\mathrm{CDCl}_{3}\right), 16: \delta 4.62\left(\mathrm{~s}, 2 \mathrm{H}, \mathrm{CH}_{2} \mathrm{CO}\right), 5.33\left(\mathrm{~s}, 2 \mathrm{H}, \mathrm{CH}_{2} \mathrm{Ph}\right), 7.22-7.65(\mathrm{M}, 11 \mathrm{H}, \mathrm{ArH}), 8.06$ (s, 1H, ArH), 8.32 (s, 1H, ArH). m/z (512, 15\%); $\mathrm{IR}_{\mathrm{KBr}} \mathrm{cm}^{-1}(3075,3030,2980,2870,1695,1685$, $1600,1490,1460,1200,700) .17: \delta 4.69\left(\mathrm{~s}, 2 \mathrm{H}, \mathrm{CH}_{2} \mathrm{CO}\right), 5.32\left(\mathrm{~s}, 2 \mathrm{H}, \mathrm{CH}_{3} \mathrm{Ph}\right), 7.22-7.31(\mathrm{~m}, 6 \mathrm{H}$, ArH), 7.34-7.62 (dd, J=8.5 Hz, 4H, ArH), 8.12 (s, 1H, ArH), 8.35 (s, 1H, ArH). m/z (548, 7\%), $(546,20 \%) ; \mathrm{IR}_{\mathrm{KBr}} \mathrm{cm}^{-1}(3070,3040,2980,2870,1700,1680,1600,1490,1460,1200,1050,700)$. 18: $\delta 4.65\left(\mathrm{~s}, 2 \mathrm{H}, \mathrm{CH}_{2} \mathrm{CO}\right), 5.38\left(\mathrm{~s}, 2 \mathrm{H}, \mathrm{CH}_{2} \mathrm{Ph}\right), 7.23-7.34(\mathrm{~m}, 6 \mathrm{H}, \mathrm{ArH}), 7.35-7.60(\mathrm{~m}, 4 \mathrm{H}, \mathrm{ArH})$, $8.08(\mathrm{~s}, 1 \mathrm{H}, \mathrm{ArH}), 8.32(\mathrm{~s}, 1 \mathrm{H}, \mathrm{ArH}) . \mathrm{m} / \mathrm{z}(530,22 \%), \mathrm{IR}_{\mathrm{KBr}} \mathrm{cm}^{-1}(3060,3040,2950,2870,1701$, $1685,1601,1505,1470,1205,1070,730) .19: \delta 4.88\left(\mathrm{~s}, 2 \mathrm{H}, \mathrm{CH}_{2} \mathrm{CO}\right), 5.37\left(\mathrm{~s}, 2 \mathrm{H}, \mathrm{CH}_{2} \mathrm{Ph}\right), 7.29-$ $7.37(\mathrm{~m}, 6 \mathrm{H}, \mathrm{ArH}), 7.97-7.99(\mathrm{~m}, 1 \mathrm{H}, \mathrm{ArH}), 8.29-8.41(\mathrm{~m}, 5 \mathrm{H}, \mathrm{ArH}) . \mathrm{m} / \mathrm{z}(557,8 \%) ; \mathrm{IR}_{\mathrm{KBr}} \mathrm{cm}^{-1}$ $(3080,3050,2970,2860,1700,1685,1602,1510,1470,1220,1070,705)$. 


\section{3-Benzyl-6-iodo-2-[N-(substituted phenyl) carbamoylmethylthio]-4(3H)-quinazolinone 20-22:}

To a solution of $1(3.9 \mathrm{~g}, 0.01 \mathrm{~mol})$ in acetone $(50 \mathrm{ml})$, anhydrous $\mathrm{K}_{2} \mathrm{CO}_{3}(2.0 \mathrm{~g})$ was added followed by the appropriate $2^{\prime}$-chloro-4-substituted-acetanilide $(0.012 \mathrm{~mol})$. The reaction mixture was heated under reflux for $20 \mathrm{~h}$, then filtered while hot and the filtrate was concentrated in vacuo. The separated solid was filtered, washed with water, dried and recrystallized from the suitable solvent (Table 1). ' $\mathrm{H}$ NMR (DMSO-d $\mathrm{d}_{6}$ ), 20: $\delta 4.21$ (s, 2H, $\left.\mathrm{SCH}_{2} \mathrm{CO}\right), 5.36\left(\mathrm{~s}, 2 \mathrm{H}, \mathrm{CH}_{2} \mathrm{Ph}\right), 7.06-$ $7.65(\mathrm{~m}, 11 \mathrm{H}, \mathrm{ArH}), 8.06$ (d, $J=15 \mathrm{~Hz}, 1 \mathrm{H}, \mathrm{ArH}), 8.37$ (s, 1H, ArH), 10.38 (brs, $1 \mathrm{H}, \mathrm{NH}) . \mathrm{m} / \mathrm{z}(527$, $31 \%) ; \mathrm{IR}_{\mathrm{KBr}} \mathrm{cm}^{-1}(3300,3065,3030,2980,2880,1705,1685,1600,1490,1470,1200,700) .21: \delta$ $4.22\left(\mathrm{~s}, 2 \mathrm{H}, \mathrm{SCH}_{2} \mathrm{CO}\right), 5.38\left(\mathrm{~s}, 2 \mathrm{H}, \mathrm{CH}_{2} \mathrm{Ph}\right), 7.29-7.69$ (m, 10H, $\left.\mathrm{ArH}\right), 8.12$ (d, $\left.J=15 \mathrm{~Hz}, 1 \mathrm{H}, \mathrm{ArH}\right)$, 8.22 (brs, $1 \mathrm{H}, \mathrm{ArH}), 8.39$ (s, $1 \mathrm{H}, \mathrm{ArH}) . \mathrm{m} / \mathrm{z}(563,6 \%),(561,17 \%), \mathrm{IR}_{\mathrm{KBr}} \mathrm{cm}^{-1}(3250,3070,3040$, $2980,2800,1705,1690,1600,1500,1485,1210,730) .22: \delta 4.24\left(\mathrm{~s}, 2 \mathrm{H}, \mathrm{SCH}_{2} \mathrm{CO}\right), 5.34(\mathrm{~s}, 2 \mathrm{H}$, $\operatorname{ArH}), 7.27-7.64(\mathrm{~m}, 10 \mathrm{H}, \mathrm{ArH}), 8.14$ (d, $J=15 \mathrm{~Hz}, 1 \mathrm{H}, \mathrm{ArH}), 8.36$ (s, 1H, ArH), 8.72 (brs, 1H, NH). $\mathrm{m} / \mathrm{z}(607,23 \%),(605,24 \%) ; \mathrm{IR}_{\mathrm{KBr}} \mathrm{cm}^{-1}(3200,3060,3035,2990,2885,1705,1685,1600,1510$, $1495,1200,710)$.

\section{Bis-[3-benzyl-6-iodo-4(3H)-quinazolinone-2-yl]disulphide 23:}

A solution of $1(3.9 \mathrm{~g}, 0.01 \mathrm{~mol})$ in $10 \% \mathrm{NaOH}(30 \mathrm{ml})$ was stirred at room temperature while an iodine solution $(3.8 \mathrm{~g} / 50 \mathrm{ml} \mathrm{EtOH})$ was added dropwise. Stirring was continued overnight. The obtained solid was filtered, washed with water, dried and recrystallized from $\mathrm{CHCl}_{3} / \mathrm{Hexane}$ (Table 1). ${ }^{1} \mathrm{H}$ NMR $\left(\mathrm{CDCl}_{3}\right): \delta 5.21\left(\mathrm{~s}, 2 \mathrm{H}, \mathrm{CH}_{2} \mathrm{Ph}\right), 5.24\left(\mathrm{~s}, 2 \mathrm{H}, \mathrm{CH}_{3} \mathrm{Ph}\right), 7.13-7.46(\mathrm{~m}, 12 \mathrm{H}, \mathrm{ArH})$, 8.04 (s, 1H, ArH), 8.07 (s, 1H, ArH), 8.34 (s, 2H, ArH). m/z (786, 25\%), IR $\mathrm{KBr} \mathrm{cm}^{-1}(3070,3050$, $2970,1705,1600,1520,1480,1100,720,550)$ 


\section{1,2-Bis-[3-benzyl-6-iodo-4(3H)-quinazolinone-2-yl-thio]ethane 24:}

To a stirred solution of $1(3.9 \mathrm{~g}, 0.01 \mathrm{~mol})$ and $\mathrm{NaOH}(1.0 \mathrm{~g}, 0.025 \mathrm{~mol})$ in DMF $(50 \mathrm{ml})$, dibromoethane $(1.9 \mathrm{~g}, 0.85 \mathrm{ml}, 0.01 \mathrm{~mol})$ was added dropwise. The reaction mixture was heated under reflux for $8 \mathrm{~h}$. Upon cooling, the mixture was poured into ice water and the obtained solid was filtered, dried and recrystallized from AcOH (Table 1). ${ }^{1} \mathrm{H}$ NMR (DMSO- $\left.\mathrm{d}_{6}\right): \delta 2.10(\mathrm{t}, J=10$ $\left.\mathrm{Hz}, 2 \mathrm{H}, \mathrm{CH}_{2} \mathrm{CH}_{2}\right), 2.34\left(\mathrm{t}, J=10 \mathrm{~Hz}, 2 \mathrm{H}, \mathrm{CH}_{2} \mathrm{CH}_{2}\right), 5.34$ (s, $\left.2 \mathrm{H}, \mathrm{CH}_{2} \mathrm{Ph}\right), 5.36\left(\mathrm{~s}, 2 \mathrm{H}, \mathrm{CH}_{2} \mathrm{Ph}\right), 7.06-$ 7.39 (m, 12H, ArH), 8.24 (s, 2H, ArH), 8.56 (s, 2H, ArH). m/z (814, 27\%), IR $\mathrm{KBr}_{\mathrm{r}} \mathrm{cm}^{-1}(3080,3050$, $2980,2870,2850,1700,1605,1485,1440,1200,705)$.

\section{3-Benzyl-6-iodo-2-[2-(1-phthalimido)ethylthio]-4(3H)-quinazolinone 25:}

To a solution of $1(3.9 \mathrm{~g}, 0.01 \mathrm{ml})$ in acetone $(50 \mathrm{ml})$, anhydrous $\mathrm{K}_{2} \mathrm{CO}_{3}(2.0 \mathrm{~g})$ was added followed by $\mathrm{N}$-(2-chloroethyl)phthalimide $(2.5 \mathrm{~g}, 0.012 \mathrm{~mol})$. The reaction mixture was heated under reflux for $12 \mathrm{~h}$, filtered while hot, concentrated in vacuo and the separated solid was filtered, dried and recrystallized from AcOH (Table 1). ${ }^{1} \mathrm{H}$ NMR (DMSO-d $\mathrm{d}_{6}$ ): $\delta 2.72(\mathrm{t}, J=11 \mathrm{~Hz}, 2 \mathrm{H}$, $\left.\mathrm{CH}_{2} \mathrm{CH}_{2}\right), 3.43\left(\mathrm{t}, J=11 \mathrm{~Hz}, 2 \mathrm{H}, \mathrm{CH}_{2} \mathrm{CH}_{2}\right), 5.24\left(\mathrm{~s}, 2 \mathrm{H}, \mathrm{CH}_{2} \mathrm{Ph}\right), 7.20-7.59$ (m, $\left.10 \mathrm{H}, \mathrm{ArH}\right), 8.06(\mathrm{~s}$, $1 \mathrm{H}, \mathrm{ArH}), 8.36$ (s, 1H, ArH). m/z (567, 14\%); $\operatorname{IR}_{\mathrm{KBr}} \mathrm{cm}^{-1}(3070,3055,2980,2880,2850,1815$, $1725,1705,1600,1500,1466,1200,710)$.

\section{References}

1. Renault G.S., Renault J., Servolles P.G., Baron M., Paoletti C., Cros S., Bissery M.C., Lavella F. Atassi G. (1991), J. Med. Chem. 34: 38-46.

2. Bavetsias V., Jackman A.L., Marriott J.H., Kimbell R., Gibson W., Boyle F.T., Bisset G.M. (1997), J. Med. Chem. 40: 1495-1510.

3. Bavetsias V., Marriott J.H., Melin C., Kimbell R., Matusiak Z., Boyle F.T., Jackman A. (2000), J. Med. Chem. 43: 1910-1926. 
4. Kersemaekers A.M.F., Fleuren G.J., Kenter E.G., Van den Broek L.J., Uljee S.M., Hermans J., Van de Vijver M.J. (1999), Clin. Cancer Res. 5: 577-586.

5. Maurizi M., Almadori G., Ferrandina G., Distefano M., Romanni M.E., Cadoni G., Benedetti-Panici P., Paludetti G., Scambia G., Mancuso S. (1996), Br. J. Cancer 74: 12531257.

6. Thompson A.M., Bridges A.J., Fry D.W., Kraker A.J., Denny W.A. (1995), J. Med. Chem. 38: $3780-3788$.

7. Bridges A.J., Zhou H., Cody D.R., Rewcastle G.W., McMichael A., Showalter H.D., Fry D.W., Kraker A.J., Denny W.A. (1996), J. Med. Chem. 39: 267-276.

8. Wakeling A.E., Barker A.J., Davies D.H., Brown D.S., Green L.R., Cartlidge S.A., Woodburn J.R. (1996), Breast Cancer Res. Treat. 38: 67-73.

9. Rewcastle G.W., Palmer B.D., Thompson A.M., Bridges A.J., Cody D.R., Zhou H., Fry D.W., McMichael A., Denny W.A. (1996), J. Med. Chem. 39: 1823-1835.

10. Fry D.W., Nelson J.M., Slintak V., Keller P.R., Rewcastle G.W., Denny W.A., Zhou H., Bridges A.J. (1997), Biochem. Pharmacol. 54: 877-887.

11. Smaill J.B., Palmer B.D., Rewcastle G.W., Denny W.A., McNamara D.J., Leopold W.R., Fry D.W., Slintak V., Roberts B.J., Uincent P.W., Patmore S.J. (1999), J. Med. Chem. 42: 1803-1815.

12. Smaill J.B., Rewcastle G.W., Loo J.A., Gries K.D., Chan O.H., Reyner E.L., Lipka E., Showalter H.D., Vincent P.W., Elliot W.L., Denny W.A. (2000), J. Med. Chem. 43: 13801397.

13. Wissner A., Berger D.M., Boschelli D.H., Floyd M.B., Greenberger L.M., Tsou H., Upeslacis E., Wang Y.F., Zhang N. (2000), J. Med. Chem. 43: 3244-3256.

14. Werbel L.M., Degnan M.J. (1987), J. Med. Chem. 30: 2151-2154.

15. Gangjee A., Shi J., Queener S.F. (1997), J. Med. Chem. 40: 1930-1936.

16. Gangjee A., Vidwans A.P., Vasudevan A., Queener S.F., Kisliuk R.L., Cody V., Li R., Galitsky N., Luft J.R., Pangborn W. (1998), J. Med. Chem. 41: 3426-3434.

17. Rosowsky A., Papoulis A.T., Forsch R.A., Queener S.F. (1999), N. Mejd. Chem 42: 10071017.

18. Hayes J.D., Wolf C.R. (1990), Biochem. J. 272: 281-295.

19. Harris A.L. (1985), Int. J. Radiat. Biol. 48: 675-690.

20. Griffin R.J., Srinivasan S., Bowman K., Calvert A.H., Curtin N.J., Newell D.R., Pemberton L.C., Golding B.T. (1998). J. Med. Chem. 41: 5247-5256. 
21. El-Subbagh H.I., El-Sherbeny M.A., Nasr M.N., Goda F.E., Badria F.A. (1995), Boll. Chim. Farmaceutico 134: 80-84.

22. El-Subbagh H.I., Abu-Zaid S.M., Mahran M.A., Badria F.A., Al-Obaid A.M. (2000), J. Med. Chem. 43: 2915-2921.

23. El-Subbagh H.I., El-Naggar W.A., Badria F.A. (1994), Med. Chem. Res. 3: 505-516.

24. Al-Obaid A.M., El-Shafie F.S., Al-Mutairi M.S., El-Subbagh H.I. (1999), Sci. Pharm. 67: 129-147.

25. Abdel-Hamid S.G., El-Obeid H.A., El-Majed A.A., El-Kashef H.A., El-Subbagh H.I. (2000), Med. Chem. Res., in press.

26. Grever M., Schepartz S., Chabner B. (1992), Semin. Oncol. 19: 622-638.

27. Monks A., Scudiero D., Skehan P. (1991), J. Natl. Cancer Inst. 83: 757-766.

28. Boyd M., Paull K. (1995), Drug Dev. Res. 34: 91-109. 